\title{
NONWEAKLY COMPACT OPERATORS FROM ORDER-CAUCHY COMPLETE $C(S)$ LATTICES, WITH APPLICATION TO BAIRE CLASSES

\author{
BY
}

FREDERICK K. DASHIELL, JR.

\begin{abstract}
This paper is concerned with the connection between weak compactness properties in the duals of certain Banach spaces of type $C(S)$ and order properties in the vector lattice $C(S)$. The weak compactness property of principal interest here is the condition that every nonweakly compact operator from $C(S)$ into a Banach space must restrict to an isomorphism on some copy of $l^{\infty}$ in $C(S)$. (This implies Grothendieck's property that every $w^{*}$-convergent sequence in $C(S)^{*}$ is weakly convergent.) The related vector lattice property studied here is orderCauchy completeness, a weak type of completeness property weaker than $\sigma$-completeness and weaker than the interposition property of Seever. An application of our results is a proof that all Baire classes (of fixed order) of bounded functions generated by a vector lattice of functions are Banach spaces satisfying Grothendieck's property. Another application extends previous results on weak convergence of sequences of finitely additive measures defined on certain fields of sets.
\end{abstract}

0. Introduction. We study here $l^{\infty}$ embedding properties of a family of Banach spaces of type $C(S)$ which properly includes all $C(S)$ for $\sigma$-Stonian $S$ and all Baire classes of bounded functions. (A compact space $S$ is $\sigma$-Stonian if and only if the lattice $C(S)$ is boundedly $\sigma$-complete.) The Banach lattices studied here are called "up-down semicomplete", and satisfy the vector-lattice property that whenever a sequence $f_{1} \leqslant f_{2} \leqslant \ldots$ sits below a sequence $g_{1} \geqslant g_{2} \geqslant \ldots$ and $\wedge_{n=1}^{\infty}\left(g_{n}-f_{n}\right)$ $=0$ in the lattice $C(S)$, then $\bigvee f_{n}=\wedge g_{n}$ exists in $C(S)$ (see Definition 1.1). This property is equivalent to order-Cauchy completeness, which has been studied by Everett [21] and Papangelou [22].

$\$ 1$ gives several characterizations of these Banach lattices by topological properties of the maximal ideal space $S$ or by equivalent lattice conditions. $C(S)$ is up-down semicomplete (i.e., order-Cauchy complete) if and only if every dense open $F_{\sigma}$ set $V$ satisfies $\beta V=S$ (Theorem 1.5). The Boolean algebra $A$ is up-down semicomplete (with the obvious meaning) if and only if $C\left(S_{A}\right)$ is up-down semicomplete, where $S_{A}$ is the Stone space (Theorem 1.8).

(Much more has recently been learned about the lattice and algebra structure of these spaces, and about the topological structure of their maximal ideal spaces. See the paper [4].)

Received by the editors October 10, 1977 and, in revised form, April 3, 1980.

1980 Mathematics Subject Classification. Primary 46E15, 46E05, 46A40; Secondary 26A21, 28 A60. 
$\$ 2$ contains the main result of this paper (Theorem 2.5). It is an extension, to certain order-Cauchy complete $C(S)$ spaces (including all Baire classes), of the Grothendieck-Rosenthal theorem that $\sigma$-Stonian spaces satisfy the following property: every nonweakly compact operator from $C(S)$ into a Banach space restricts to an isomorphism on some isometric copy of $l^{\infty}$ in $C(S)$ [15, Theorem 3.7, p. 32].

This immediately yields all Baire classes (as well as a larger class of functionlattices) as new examples of Banach spaces which satisfy Grothendieck's propertynamely that $w^{*}$ convergence for sequences in the dual implies weak convergence (see Corollary to Theorem 3.5). In this sense, Baire classes have some isomorphic resemblance, as Banach spaces, to $C(S)$ for $\sigma$-Stonian $S$. This is contrasted with the fact that the Baire classes (of countable order) on an uncountable Polish space are not even linearly isomorphic to a complemented subspace of any $C(S)$ for $\sigma$-Stonian $S$, and their maximal ideal spaces fail even to be $F$-spaces in a rather strong way (these facts are proved in [2]). The methods of $\$ 2$ yield results for weak convergence and uniform boundedness of sequences of finitely additive measures on certain order-Cauchy complete Boolean algebras, again generalizing known results for the $\sigma$-complete case, i.e., $\sigma$-fields of sets (see Theorem 2.6).

$\$ 3$ is devoted to application of the above results to a large class of examples. We describe a family of Baire-type vector sublattices $L$ of $\mathbf{R}^{X}$ (for an abstract set $X$ ), which can be represented as $C(S)$ spaces satisfying the conditions of $\$ 2$ (Theorem 3.4). The important property of these $L$ is that they satisfy a pointwise version of up-down semicompleteness (Definition 3.1), and furthermore the lattice and pointwise countable suprema are the same. These lattices include all Baire classes (of fixed order) of bounded functions generated by sequential pointwise limits from a vector sublattice containing 1 (Theorem 3.5). These results yield information about weak convergence and uniform boundedness of sequences of finitely additive measures on certain fields of sets, generalizing Phillips' lemma and other known results for $\sigma$-fields (see Theorem 3.6).

Terminology and notation. For a set $S$ and a mapping $f: S \rightarrow \mathbf{R},[f>r]$ denotes $\{x \in S: f(x)>r\}, r \in \mathbf{R}$; similarly for $[f=r]$, etc; $\operatorname{coz}(f)=[f \neq 0]$ is the "cozero set of $f$ ". If $S$ is a topological space, then $C(S)$ is the space of all continuous $\mathbf{R}$-valued functions on $S$, a cozero set in $S$ is $\operatorname{coz}(f)$ for some $f \in C(S)$ (equivalently, for some bounded $f \in C(S)$ ); a zero set in $S$ is the complement of a cozero set. The zero sets are just the sets $f^{-1}(K)$ for closed $K \subset \mathbf{R}$ and $f \in C(S)$; the cozero sets are the $f^{-1}(G)$ for open $G \subset \mathbf{R}$ and $f \in C(S)$. In a normal space $S$, these are just the closed $G_{\delta}$ sets and the open $F_{\sigma}$ sets, respectively. We use these facts often without further mention. The Baire sets of a space are the sets in the $\sigma$-field generated by the zero sets. A subspace $G$ of a space $S$ is $C^{*}$-embedded if every bounded $f \in C(G)$ extends continuously to $S$. The standard reference for all this is [7].

1. Order-Cauchy complete lattices. The material which follows concerns both vector lattices and Boolean algebras. The property studied in this section is a weak type of sequential completeness which can be defined in two equivalent ways. These definitions appear in (c) and (d) below. 
Definition 1.1. Suppose $L$ is a vector lattice or a Boolean algebra.

(a) A sequence $\left\{f_{n}\right\}$ in $L$ is called order-Cauchy if there exists a decreasing sequence $u_{1} \geqslant u_{2} \geqslant \ldots$ in $L$ with $\wedge u_{n}=0$ in $L$ and $\left|f_{n}-f_{n+m}\right| \leqslant u_{n}$ for all $n, m=1,2,3, \ldots$ (For a Boolean algebra, substitute $f_{n} \Delta f_{n+m}<u_{n}$.)

(b) A sequence $\left\{f_{n}\right\}$ in $L$ order-converges to $g$ in $L$ if there exists a decreasing sequence $u_{1} \geqslant u_{2} \geqslant \ldots$ in $L$ with $\wedge u_{n}=0$ in $L$ and $\left|f_{n}-g\right| \leqslant u_{n}$ for all $n=1,2,3, \ldots$ (For a Boolean algebra, substitute $f_{n} \Delta g \leqslant u_{n}$.)

(c) $L$ is called order-Cauchy complete if every order-Cauchy sequence in $L$ order-converges in $L$ to some element of $L$.

(d) $L$ is called up-down semicomplete if, whenever an increasing sequence $f_{1} \leqslant f_{2}$ $\leqslant \ldots$ sits below a decreasing sequence $g_{1} \geqslant g_{2} \geqslant \ldots$ such that $\wedge_{n=1}^{\infty}\left(g_{n}-f_{n}\right)$ $=0$ in $L$, then $\bigvee f_{n}=\wedge g_{n}$ exists in $L$.

REMARK. With respect to (d), in the presence of the requirement $\wedge\left(g_{n}-f_{n}\right)=$ 0 , the existence of either $\bigvee f_{n}$ or $\wedge g_{n}$ is equivalent to the existence of both (and then they are equal), and this is equivalent to the existence of some $h \in L$ such that $f_{n} \leqslant h \leqslant g_{n}, n=1,2,3, \ldots$.

The equivalence of the definitions in (c) and (d) is not immediately obvious. In fact, the property of up-down semicompleteness is much easier to test in examples than order-Cauchy completeness, and it resembles some related interposition properties of vector lattices which have been previously studied (e.g., by Seever [18]). This paper is based entirely on definition (d), and was motivated largely on the observation that the functions of a fixed Baire class form a vector lattice which is up-down semicomplete (see Theorem 3.5). The equivalence of this property to order-Cauchy completeness is not essential to this paper. Order-Cauchy completeness was introduced by Everett [21], and his work was extended by Papangelou [22]. Both used different names for the concept; the present name occurs in Quinn [23]. Its equivalence with up-down semicompleteness follows immediately from the following lemma of Papangelou [22, 2.10]:

Proposition 1.2. A sequence $\left\{f_{n}\right\}$ in $L$ is order-Cauchy if and only if there exist an increasing sequence $g_{1} \leqslant g_{2} \leqslant \ldots$ and a decreasing sequence $h_{1} \geqslant h_{2} \geqslant \ldots$ such that $g_{n} \leqslant f_{n} \leqslant h_{n}$ for all $n$ and $\bigwedge\left(h_{n}-g_{n}\right)=0$.

As a corollary, we obtain the following fact.

Proposition. $L$ is up-down semicomplete if and only if $L$ is order-Cauchy complete.

In this paper we shall usually use the terminology order-Cauchy complete. However, the reader should bear in mind that operationally the property is being used in the form of up-down semicompleteness.

We now characterize the order-Cauchy complete vector lattices $C(S)$ (for compact $S$ ), and the up-down semicomplete Boolean algebras, by simple topological conditions on the maximal ideal space and by some equivalent lattice conditions. One characterization is that every dense cozero set of $S$ should be $C^{*}$ embedded (see (4) of Theorem 1.5 below). It turns out that these compact spaces $S$ coincide with 
certain maximal ideal spaces which arise in another context: namely, in the representation of certain abstract algebras as spaces of continuous, almost-everywhere finite, extended real-valued functions on $S$. See Henriksen and Johnson [12, Proposition 2.2]. Completely regular spaces $S$ such that every dense cozero set is $C^{*}$-embedded have been dubbed quasi-F spaces in the paper [4] (which in some ways is a sequel to this section), and their properties are rather extensively studied there.

The characterization given here is in the same spirit as the characterization, due to Seever [18], of vector lattices and Boolean algebras which satisfy the following stronger interposition property: for every increasing sequence $f_{1} \leqslant f_{2}<\ldots$ sitting below a decreasing sequence $g_{1} \geqslant g_{2} \geqslant \ldots$, there exists $h$ such that $f_{n}<h \leqslant g_{n}$, $n=1,2,3, \ldots$ For these, the appropriate dual topological property on the maximal ideal space $S$ turned out to be that of a compact $F$-space: each two disjoint open $F_{\sigma}$ sets in $S$ have disjoint closures. The analogous topological property for order-Cauchy complete $C(S)$ is (11) of Theorem 1.8 below. Compact $F$-spaces are also characterized by the property that every cozero set is $C^{*}$ embedded [7, p. 208]; or that for each pair $V, W$ of cozero sets, $\overline{V-W} \cap \overline{W-V}=\varnothing$; or that for each pair $V, W$ of cozero sets, $\overline{V \cap W}=\bar{V} \cap \bar{W}$. (The proofs of these last two are not difficult and proceed exactly as below.) The analogous properties for order-Cauchy complete $C(S)$ appear in (4) and (5) of Theorem 1.5, and in (12) of Theorem 1.8, respectively. The analogy with $F$-spaces goes farther. There are algebraic and additional vector-lattice characterizations, in terms of principal ideals and absolutely convex (i.e., "solid") ideals; see the article [4] for details.

We start with a lemma which is similar to a lemma of Aronszajn and Panitchpakdi (see [1, p. 432, Lemma 1]).

LEMMA 1.3. Suppose $S$ is a normal topological space and $f, g$ are any two bounded functions on $S$ with $f \leqslant g$ on $S$. In order that there exist $h \in C(S)$ such that $f \leqslant h \leqslant g$ on $S$, it is necessary and sufficient that for each $a<b$ in $\mathbf{R}$, the disjoint sets $[f>b]$ and $[g<a]$ have disjoint closures in $S$.

Proof. For any bounded $h: S \rightarrow \mathbf{R}$, define the upper and lower envelopes by

$$
h^{*}(x)=\inf _{N(x)} \sup _{t \in N(x)} h(t), \quad h_{*}(x)=\sup _{N(x)} \inf _{t \in N(x)} h(t),
$$

$x \in S$, where $N(x)$ runs through a neighborhood base at $x$. Then $h^{*}$ is upper semicontinuous on $S$ and $h_{*}$ is lower semicontinuous on $S$, and $h_{*} \leqslant h \leqslant h^{*}$ on $S$.

By the Hahn-Tong interposition theorem [19, p. 100], if $f^{*} \leqslant g_{*}$, then there exists $h \in C(S)$ such that $f^{*} \leqslant h \leqslant g_{*}$. Conversely, if $f \leqslant h \leqslant g$ for some $h \in C(S)$, then obviously $f^{*} \leqslant h^{*}=h=h_{*} \leqslant g_{*}$ on $S$. Thus a continuous $h$ exists between $f$ and $g$ if and only if $f^{*} \leqslant g_{*}$. But $f^{*}\left(x_{0}\right)>g_{*}\left(x_{0}\right)$ for some $x_{0} \in S$ if and only if for some $a<b$ in $\mathbf{R}, x_{0} \in \overline{[f>b]} \cap \overline{[g<a]}$, and the lemma is proved.

We offer in passing an alternative, short proof of Seever's theorem. Seever used a Urysohn type of construction which, in the present proof, gets absorbed into the above lemma in the form of the Hahn-Tong Theorem. 
Corollary 1.4 (Seever [18]). If $S$ is a compact $F$-space and $f_{1} \leqslant f_{2} \leqslant \ldots \leqslant g_{2}$ $\leqslant g_{1}$ in $C(S)$ then there exists $h \in C(S)$ such that $f_{n} \leqslant h \leqslant g_{n}, n=1,2,3, \ldots$

Proof. If $f(x)=\lim f_{n}(x)$ and $g(x)=\lim g_{n}(x), x \in S$, then $f<g$ on $S$. If $a<b$ then $[f>b]=\bigcup_{n=1}^{\infty}\left[f_{n}>b\right]$ and $[g<a]=\bigcup_{n=1}^{\infty}\left[g_{n}<a\right]$ are disjoint open $F_{\sigma}$ sets, so they have disjoint closures by assumption. Lemma 1.3 produces the desired $h$.

TheORem 1.5. Suppose $S$ is compact, and let $L$ denote the vector lattice $C(S)$. The following (1)-(5) are equivalent:

(1) $L$ is order-Cauchy complete (equivalently, $L$ is up-down semicomplete);

(2) for every order-Cauchy sequence $\left\{f_{n}\right\}$ in $L, \bigvee_{n=1}^{\infty} f_{n}$ exists in $L$;

(3) if $f_{n} \wedge f_{m}=0$ for $m \neq n$ and order-lim $f_{n}=0$, then $\bigvee_{n=1}^{\infty} f_{n}$ exists in $L$;

(4) every dense cozero set in $S$ is $C^{*}$ embedded;

(4') for every dense cozero set $V \subset S, \beta V=S$;

(5) if $V$ and $W$ are cozero sets with $V \cup W$ dense in $S$, then $\overline{V-W} \cap \overline{W-V}=$ $\varnothing$.

Furthermore, conditions (1)-(5) imply:

(6) if $f_{n} \wedge f_{m}=0$ in $L$ for $n \neq m$ and $\bigvee_{n=1}^{\infty} f_{n}$ exists in $L$, then $\bigvee_{i=1}^{\infty} f_{n_{i}}$ exists in $L$ for every subsequence $n_{1}<n_{2}<\ldots$

Proof. $(1) \Leftrightarrow(2)$. This is true for any lattice group and is due to Everett [21, p. 112]. See also Papangelou [22, 2.13].

(2) $\Rightarrow(3)$. Clear.

(3) $\Rightarrow$ (6). Suppose $f_{n} \wedge f_{m}=0$ for $n \neq m$, and $\bigvee_{n=1}^{\infty} f_{n}=h$ exists. Let $g_{1}=h$ and $g_{n+1}=h-\sum_{i=1}^{n} f_{i}=h-\left(f_{1} \vee \cdots \vee f_{n}\right), n=1,2, \ldots$ Then $f_{n} \leqslant g_{n}, n=$ $1,2,3, \ldots$, and $g_{1} \geqslant g_{2} \geqslant \ldots$ with $\bigwedge_{n=1}^{\infty} g_{n}=0$. For any subsequence, $f_{n_{i}}<g_{n_{i}}$ and $\wedge_{i=1}^{\infty} g_{n_{i}}=0$, so by (3), $\bigvee_{i=1}^{\infty} f_{n_{i}}$ exists. This proves (6).

$(3) \Rightarrow(5)$. The idea for this part of the proof derives from a disjointness argument of Veksler and Geiler [20, Proposition 2, p. 31]. Suppose $V$ and $W$ are cozero sets in $S$ with $V \cup W$ dense in $S$. We shall construct $f \in C(S)$ so that $f$ is 1 on $V-W$ and 0 on $W-V$. Write $V=\cup_{n=1}^{\infty} V_{n}, W=\cup_{n=1}^{\infty} W_{n}$ where the $V_{n}$ and $W_{n}$ are open and $\bar{V}_{n} \subset V_{n+1}, \bar{W}_{n} \subset W_{n+1}, n=1,2,3, \ldots$ Set $V_{0}=V_{-1}=\varnothing$. Define for each $n, h_{n}$ and $g_{n}^{\prime}$ in $C(S)$ so that $0 \leqslant h_{n} \leqslant 1,0 \leqslant g_{n}^{\prime} \leqslant 1$, and

$$
\begin{aligned}
& h_{n}=1 \text { on } \bar{V}_{n}-V_{n-1} \text {, } \\
& =0 \text { on } \bar{V}_{n-2} \cup V_{n+1}^{c} \text {, } \\
& g_{n}^{\prime}=1 \text { on } W^{c} \cap V_{n-1}^{c} \text {, } \\
& =0 \text { on } \bar{V}_{n-2} \cup \bar{W}_{n} \text {. }
\end{aligned}
$$

Now put $g_{n}=g_{1}^{\prime} \wedge \cdots \wedge g_{n}^{\prime}, f_{n}=h_{n} \wedge g_{n}, n=1,2,3, \ldots$ If $|m-n| \geqslant 3$, then $h_{n} \wedge h_{m}=0$, so for $i=0,1,2$, the sequences $\left\{f_{3 n-i}\right\}_{n=1}^{\infty}$ consist of pairwise disjoint functions. Clearly $g_{1} \geqslant g_{2} \geqslant \ldots$ and for $x \in V \cup W$, eventually $g_{n}(x)=0$. Thus $\bigwedge_{n=1}^{\infty} g_{n}=0$ in $C(S)$ since $V \cup W$ is dense in $S$. Therefore, assuming (3), $u_{i}=\bigvee_{n=1}^{\infty} f_{3 n-i}$ exists for $i=0,1,2$. Set $f=u_{0} \vee u_{1} \vee u_{2}$. If $x \in V-W$, then 
$x \in \bar{V}_{n_{0}}-V_{n_{0}-1}$ for some $n=n_{0}$, so $h_{n_{0}}(x)=1$. Also, for all $n \leqslant n_{0}, x \in W^{c} \cap$ $V_{n-1}^{c}$, so $g_{n}^{\prime}(x)=1$ for $n \leqslant n_{0}$, i.e., $g_{n_{0}}(x)=1$. Thus $1=f_{n_{0}}(x) \leqslant f(x)$, so $f=1$ on $V-W$. Finally, if $y \in W-V$, then $y \in W_{n_{1}}$ for some $n=n_{1}$. Notice that all $f_{n} \equiv 0$ on the open neighborhood $G=W_{n_{1}}-\bar{V}_{n_{1}}$ of $y$ : if $n<n_{1}$ then $h_{n}=0$ on $V_{n+1}^{c} \supset \bar{V}_{n_{1}}^{c} \supset G$, and if $n \geqslant n_{1}$ then $g_{n}^{\prime}=0$ on $W_{n} \supset W_{n_{1}} \supset G$. Thus $f(y)=0$, and $f \equiv 0$ on $W-V$. Thus $\overline{V-W} \cap \overline{W-V}=\varnothing$. This proves (5).

(5) $\Leftrightarrow(4)$. Recall that a dense set $G \subset S$ is $C^{*}$ embedded if and only if each two disjoint zero subsets of $G$ have disjoint closures in $S$ (see [7, p. 84]). To verify (4) $\Rightarrow(5)$, choose cozero sets $V$ and $W$ so that $G=V \cup W$ is dense. Assuming (4), $G$ is $C^{*}$ embedded, so that the disjoint zero sets $G-V$ and $G-W$ in $G$ have disjoint closures in $S$, i.e., $\overline{V-W} \cap \overline{W-V}=\varnothing$. Conversely, for (5) $\Rightarrow$ (4) we need a simple fact: if $G$ is a cozero set in a space $S$, then every cozero subset of $G$ is a cozero subset of $S$. (Indeed, let $G=\operatorname{coz}(g), g \in C(S)$, and let $f$ be a bounded continuous function on $G$. Define $h(x)=g(x) f(x)$ for $x \in G, h(x)=0$ for $x \in S$ $-G$. Then $h \in C(S)$ and $\operatorname{coz}(h)=\operatorname{coz}(f)$, i.e., $\operatorname{coz}(f)$ is a cozero subset of $S$.) To prove $(5) \Rightarrow(4)$, let $G$ be a dense cozero set in $S$. If $Z_{1}, Z_{2}$ are disjoint zero sets in $G$, then the sets $V=G-Z_{1}$ and $W=G-Z_{2}$, being cozero subsets of $G$, are cozero subsets of $S$. But $V \cup W=G$ is dense in $S, V-W=Z_{2}$, and $W-V=$ $Z_{1}$. Assuming (5) we obtain $\bar{Z}_{1} \cap \overline{Z_{2}}=\varnothing$, so that $G$ is $C^{*}$ embedded by the above criterion.

$(4) \Leftrightarrow\left(4^{\prime}\right)$ is an immediate consequence of the definition and uniqueness of the Stone-Čech compactification [7, p. 86].

$(5) \Rightarrow(1)$. Choose $f_{1} \leqslant f_{2} \leqslant \cdots \leqslant g_{2} \leqslant g_{1}$ in $C(S)$ with $\wedge_{n=1}^{\infty}\left(g_{n}-f_{n}\right)=0$ in the lattice $C(S)$. Put $f(x)=\lim _{n} f_{n}(x), g(x)=\lim _{n} g_{n}(x)$ for $x \in S$. Then $f<g$ on $S$, and it suffices to find $h \in C(S)$ with $f \leqslant h \leqslant g$. We will use Lemma 1.3.

For each $k=1,2, \ldots$, the set $E_{k}=[g-f<1 / k]=\cup_{n=1}^{\infty}\left[g_{n}-f_{n}<1 / k\right]$ is open. Also, $E_{k}$ is dense, for otherwise there would exist $e \in C(S)$ with $\|e\|=$ $1 / k$ and $0 \leqslant e \leqslant g-f \leqslant g_{n}-f_{n}$ for all $n$, contradicting $\bigwedge_{n=1}^{\infty}\left(g_{n}-f_{n}\right)=0$. By the category theorem, $E=\bigcap_{k=1}^{\infty} E_{k}$ is dense, and $f=g$ on $E$.

Now pick $a<b$ in $\mathbf{R}$, and define

$$
V=[f>a]=\bigcup_{n=1}^{\infty}\left[f_{n}>a\right], \quad W=[g<b]=\bigcup_{n=1}^{\infty}\left[g_{n}<b\right] .
$$

Then $V$ and $W$ are cozero sets since $\left[f_{n}>a\right]$ and $\left[g_{n}<b\right]$ are. Similarly, the sets $[f>b]$ and $[g<a]$ are cozero sets, hence open. Clearly $E=[f=g] \subset V \cup W$, so $V \cup W$ is dense. Also, $E \cap[f>b] \subset V-W, E \cap[g<a] \subset W-V$. But $E$ is dense and $[f>b]$ is open, so

$$
\overline{[f>b]}=\overline{E \cap[f>b]} \subset \overline{V-W} \text {. }
$$

Similarly,

$$
\overline{[g<a]}=\overline{E \cap[g<a]} \subset \overline{W-V} \text {. }
$$

Assuming condition (5), we obtain $\overline{V-W} \cap \overline{W-V}=\varnothing$, so $[f>b] \cap[g<a]=$ $\varnothing$. By Lemma 1.3, there exists $h \in C(S)$ such that $f \leqslant h \leqslant g$. This proves (1), and concludes the proof of the theorem. 
REMARK 1.6. The implications (1) $\Leftrightarrow(2) \Rightarrow(3) \Rightarrow(6)$ are valid in every vector lattice $L$. The proofs are exactly the same as given above. As for the converses, we close the loop in Theorem 1.8 below and show that $(6) \Rightarrow(1)$ if $L=C(S)$ for $S$ compact and totally disconnected. For more general $S$, this converse is unknown. If $S$ is not assumed compact, but is only completely regular, then Theorem 1.5 and the present proof remain valid with minor modifications. Another proof for this more general case is given in [4].

COROllary 1.7. If $C(S)$ is order-Cauchy complete, then every nowhere dense zero-set of $S$ is an F-space.

Proof. If $Z \subset S$ is a nowhere dense zero set, then $V=Z^{c}$ is a dense cozero set, so $Z=S-V=\beta V-V$ by $\left(4^{\prime}\right)$ of Theorem 1.5. But $\beta V-V$ is $F$-space whenever $V$ is locally compact and $\sigma$-compact [7, p. 210].

We now turn to Boolean algebras. Without loss of generality, we may as well consider totally disconnected compact spaces $S$.

THEOREM 1.8. Suppose $S$ is a totally disconnected compact space, $L=C(S)$, and $A$ is the Boolean algebra of all closed-open sets in S. Then the following conditions are all equivalent to each other and to each of the conditions of Theorem 1.5, including (6):

(1) (repeated for emphasis) the vector lattice $L=C(S)$ is order-Cauchy complete;

(7) the Boolean algebra $A$ is up-down semicomplete (equivalently, order-Cauchy complete);

(8) if $F_{n} \leqslant G_{n}, G_{1} \geqslant G_{2} \geqslant \ldots$ and $\bigwedge_{n=1}^{\infty} G_{n}=\varnothing$ in $A$, then $\bigvee_{n=1}^{\infty} F_{n}$ exists in A ;

(9) if $F_{n} \leqslant G_{n}, F_{m} \wedge F_{n}=\varnothing$ for $m \neq n, G_{1} \geqslant G_{2} \geqslant \ldots$, and $\wedge_{n=1}^{\infty} G_{n}=\varnothing$, then $\bigvee_{n=1}^{\infty} F_{n}$ exists in $A$;

(10) if $F_{n} \wedge F_{m}=\varnothing$ in $A$ for $m \neq n$ and $\bigvee_{n=1}^{\infty} F_{n}$ exists in $A$, then $\bigvee_{i=1}^{\infty} F_{n_{i}}$ exists in $A$ for every subsequence $n_{1}<n_{2}<\cdots$;

(11) if $V$ and $W$ are disjoint cozero sets in $S$ with $V \cup W$ dense in $S$, then $\bar{V} \cap \bar{W}=\varnothing$

(12) if $V$ and $W$ are cozero sets in $S$ with $V \cup W$ dense in $S$, then $\overline{V \cap W}=\bar{V} \cap$ $\bar{W}$.

Proof. The proof goes $(1) \Rightarrow(7) \Rightarrow(8) \Rightarrow(9) \Rightarrow(10) \Rightarrow(11) \Rightarrow(5) \Rightarrow(12) \Rightarrow$ (11) and $(6) \Rightarrow(10)$. Together with Theorem 1.5 , this establishes the equivalence of all 12 properties when $S$ is totally disconnected.

$(1) \Rightarrow(7)$. Consider characteristic functions $1_{F} \in C(S)$ for $F \in A$.

$(7) \Rightarrow(8) \Rightarrow(9) \Rightarrow(10)$. These conditions are the Boolean algebra equivalents of (1), (2), (3), and (6) of Theorem 1.5. Upon embedding $A$ into $C(S)$ by characteristic functions, one uses the same proofs as given there to obtain the corresponding results for $A$.

$(10) \Rightarrow(11)$. Suppose $V$ and $W$ are disjoint open $F_{o}$ sets with $V \cup W$ dense in $S$. Since $S$ is totally disconnected, we can write $V=\cup_{n=1}^{\infty} F_{2 n-1}, W=\bigcup_{n=1}^{\infty} F_{2 n}$, where the $F_{n}, n=1,2, \ldots$, are pairwise disjoint clopen sets. Since $V \cup W$ is 
dense, then $\bigvee_{n=1}^{\infty} F_{n}$ exists (and equals $S$ ). Assuming (10), we deduce that $\bigvee_{n=1}^{\infty} F_{2 n-1}$ and $\bigvee_{n=1}^{\infty} F_{2 n}$ exist in $A$. That is, $\bar{V}=\left[\bigcup_{n=1}^{\infty} F_{2 n-1}\right]^{-}$and $\bar{W}=$ $\left[\cup_{n=1}^{\infty} F_{2 n}\right]^{-}$are open and therefore disjoint. This proves (11).

$(11) \Rightarrow(5)$. Suppose $V$ and $W$ are open $F_{\sigma}$ sets in $S$. Since $S$ is totally disconnected, $V$ and $W$ can be "reduced" by open $F_{\sigma}$ sets $V_{1}$ and $W_{1}$-i.e., there exist open $F_{\sigma}$ sets $V_{1} \subset V$ and $W_{1} \subset W$ such that $V_{1} \cap W_{1}=\varnothing$ and $V_{1} \cup W_{1}=V \cup$ $W$. This fact is a form of Kuratowski's "reduction property" in abstract set theory; see [13, Theorem 1, p. 127]. If $V \cup W$ is dense and (11) holds, then $\bar{V}_{1} \cap \bar{W}_{1}=\varnothing$, so that $\overline{V-W} \cap \overline{W-V} \subset \bar{V}_{1} \cap \bar{W}_{1}=\varnothing$. This proves (5).

(5) $\Rightarrow$ (12) follows from the relation $\bar{V} \cap \bar{W}-\overline{V \cap W} \subset \overline{V-W} \cap \overline{W-V}$ (valid for arbitrary $S$ ).

$(12) \Rightarrow(11)$ is obvious.

$(6) \Rightarrow(10)$. Consider characteristic functions.

The theorem is proved.

Remark 1.9. Conditions (5), (11), and (12) are purely topological, and the implications $(5) \Rightarrow(12) \Rightarrow(11)$ are valid in every space $S$. The converse implications do not hold in an arbitrary compact space; see [4, Example 5.6].

There are nontrivial, naturally occurring examples of totally disconnected spaces $S$ such that $C(S)$ is order-Cauchy complete, yet $S$ is not $\sigma$-Stonian or even an $F$-space. One such $S$ is the Stone space of the Boolean algebra of "ambiguous class $\alpha$ " Baire sets in any uncountable Polish space $X$; equivalently, $S$ is the maximal ideal space of the Baire class $\alpha$ functions on $X$ (for any countable ordinal $\alpha>0$ ). For proofs, see $\S 3$, where a rather more extensive class of examples is described, and $[2, \S 3]$ for failure of the $F$-property. In fact, it is the study of these Baire classes which leads to the results presented here.

2. Embedding $l^{\infty}$ into $C(S)$. In Theorem 2.5 of this section we extend, to certain order-Cauchy complete $C(S)$ lattices, the Grothendieck-Rosenthal theorem that if $S$ is $\sigma$-Stonian and $T$ is a nonweakly compact operator from $C(S)$ into a Banach space, then $T$ restricts to an isomorphism on some isometric copy of $l^{\infty}$ in $C(S)$. (See [15, Theorem 3.7, p. 32]. Compact spaces $S$ such that the lattice $C(S)$ is Dedekind $\sigma$-complete are called $\sigma$-Stonian, or basically disconnected, and are characterized by the property that each cozero set has open closure [17, p. 107].)

The major effort of the proof here is in embedding $l^{\infty}$ into $L=C(S)$. It seems that the single assumption that $L$ be order-Cauchy complete is not enough, although I know of no counterexample. However, it suffices to impose an additional condition on certain elements of the dual $L^{*}$ : for every nonzero sequentially order continuous (defined below) $\mu \geqslant 0$ in $L^{*}$, and every $f \geqslant 0$ in $L$ with $\mu(f)=0$, there exists $g \in L$ such that $g \wedge f=0$ and $\mu(g)>0$. This is a pure vector-lattice property, and does not refer explicitly to the maximal ideal space. As will be seen in the proof of Theorem 2.5, the effect of this condition is to force the action into the ideal of "singular" measures. This condition is equivalent to the requirement that every sequentially order continuous functional should be $\left(O^{+}\right)$-linear in the sense of Veksler [24]. 
This condition is nevertheless satisfied by many examples, including all $\sigma$ Stonian spaces (see Lemma 2.3) and the Baire classes discussed in the next section. However, the condition unfortunately eliminates some $F$-spaces (see Example 2.4), so the following question remains open: for every $F$-space $S$, must $C(S)$ contain $l^{\infty}$ ? A positive answer would follow from Theorem 3.5 if there exists a nonweakly compact operator from the first Baire class of $S$ into $C(S)$. The answer is positive under the assumption of the continuum hypothesis; see [15, Remark 1, p. 33].

A functional $\mu \geqslant 0$ in $C(S)^{*}$ is sequentially order continuous if $\mu\left(f_{n}\right) \rightarrow 0$ whenever $f_{1} \geqslant f_{2} \geqslant \ldots$ and $\bigwedge_{n=1}^{\infty} f_{n}=0$ in the lattice $C(S)$. We distinguish between a functional $\mu$ in $C(S)^{*}$ and the corresponding regular Borel measure, which is denoted by $\hat{\mu}$. The sequentially order continuous functionals are quite similar to the order-continuous functionals in $C(S)^{*}$, where decreasing nets replace sequences, and whose corresponding measures are called normal measures. The normal measures $\hat{\mu} \geqslant 0$ are characterized by the condition that $\hat{\mu}(E)=0$ for every meager Borel set $E \subset S[19$, p. 341]. We give a similar characterization of the sequentially order continuous functionals (whose measures might be called " $\sigma$ normal").

Proposition 2.1. For compact $S$ and $\mu \geqslant 0$ in $C(S)^{*}, \mu$ is sequentially order continuous if and only if $\hat{\mu}(E)=0$ for every meager Baire set $E \subset S$ (equivalently, $\hat{\mu}(F)=0$ for every nowhere dense zero set $F \subset S)$.

Proof. If $\mu \geqslant 0$ is sequentially order continuous and $F$ is a nowhere dense zero set, then $F=\cap{ }_{n=1}^{\infty} G_{n}$ where $G_{1} \supset G_{2} \supset \ldots$ are open. Choose $f_{n} \in C(S)$ so that $0 \leqslant f_{n} \leqslant 1, f_{n}(F)=1, f_{n}\left(G_{n}^{c}\right)=0$, and $f_{1} \geqslant f_{2} \geqslant \ldots$ Then $\wedge f_{n}=0$ and $\hat{\mu}(F) \leqslant$ $\mu\left(f_{n}\right) \rightarrow 0$, so $\hat{\mu}(F)=0$. For an arbitrary meager Baire set $E, \hat{\mu}(E)$ is approximated by $\hat{\mu}(F)$ for zero sets $F \subset E[16$, p. 305], so $\hat{\mu}(E)=0$.

Conversely, if $\hat{\mu}(F)=0$ for all nowhere dense zero sets $F$, and $f_{1} \geqslant f_{2} \geqslant \ldots \geqslant 0$ with $\wedge f_{n}=0$, let $f(x)=\lim f_{n}(x), x \in S$. Then $\mu\left(f_{n}\right)=\int f_{n} d \hat{\mu} \rightarrow \int f d \hat{\mu}$ by the monotone convergence theorem. If $\int f d \hat{\mu}>0$, then $\hat{\mu}([f \geqslant 1 / m])>0$ for some $m$, and by regularity there exists a zero set $F \subset[f \geqslant 1 / m]$ with $\hat{\mu}(F)>0$. But any such zero set $F$ is nowhere dense, since $\wedge f_{n}=0$ implies $[f \geqslant 1 / m]$ has no interior. Thus $\hat{\mu}(F)=0$, so $\int f d \hat{\mu}=\lim \mu\left(f_{n}\right)=0$, and $\mu$ is sequentially order continuous. This proves the proposition.

LEMMA 2.2. For $\mu \geqslant 0$ in $C(S)^{*}$, these are equivalent:

(1) for every $f \in C(S)$ with $f \geqslant 0$ and $\mu(f)=0$, there exists $g \in C(S)$ such that $g \wedge f=0$ and $\mu(g)>0$;

(2) the support of $\hat{\mu}$ is not contained in the boundary of any cozero set of $S$.

Proof. (1) $\Rightarrow(2)$. If $V=\operatorname{coz}(f)$ is a cozero set for $f \geqslant 0$ in $C(S)$ and $\hat{\mu}(V)>0$, then $\operatorname{supp}(\hat{\mu}) \nsubseteq \partial V$. If $\hat{\mu}(V)=0$, then assuming (1) there exists $g \in C(S)$ such that $g \wedge f=0$ and $\mu(g)>0$. But $\operatorname{coz}(g) \cap V=\varnothing$, so $\operatorname{coz}(g) \cap \partial V=\varnothing$. Since $\operatorname{supp}(\hat{\mu}) \cap \operatorname{coz}(g) \neq \varnothing, \operatorname{supp}(\hat{\mu}) \notin \partial V$.

(2) $\Rightarrow(1)$. If $f \geqslant 0$ in $C(S)$ and $\mu(f)=0$, then $\operatorname{supp}(\hat{\mu}) \cap \operatorname{coz}(f)=\varnothing$. Thus by (2) there is a point $x \in \operatorname{supp}(\hat{\mu})-\overline{\operatorname{coz}(f)}$. If $g \in C(S)$ with $0<g<1, g(x)=1$, $g=0$ on $\operatorname{coz}(f)$, then $\mu(g)>0$ and $f \wedge g=0$. This proves the lemma. 
LEMMA 2.3. If $S$ is a compact $\sigma$-Stonian space, then for every nonzero sequentially order continuous $\mu \geqslant 0$ in $C(S)^{*}$ and every $f \geqslant 0$ such that $\mu(f)=0$, there exists $g \in C(S)$ such that $g \wedge f=0$ and $\mu(g)>0$.

Proof. If $\mu \geqslant 0$ is sequentially order continuous and $V$ is a cozero set, then $\bar{V}$ is open so $\partial V=\bar{V}-V$ is a nowhere dense zero set. Thus $\hat{\mu}(\partial V)=0$ by Proposition 2.1, so if $\mu \neq 0$ then $\operatorname{supp}(\hat{\mu}) \nsubseteq \partial V$. The result follows from Lemma 2.2.

EXAmPle 2.4. For the $F$-space $S=\beta \mathbf{N}-\mathbf{N}$ [7, p. 210], every $\mu \geqslant 0$ in $C(S)^{*}$ is sequentially order continuous, but many of them have their supports in the boundary of some cozero set. Indeed, every nonempty Baire set in $\beta \mathbf{N}-\mathbf{N}$ has nonempty interior (because every Baire set in any space is a union of zero sets, and every nonempty $G_{\delta}$ set in $\beta N-N$ has nonempty interior [7, §6S(8), p. 99]). Thus every $\mu \geqslant 0$ in $C(\beta \mathbf{N}-\mathbf{N})^{*}$ is sequentially order continuous by Proposition 2.1 . But for every nonclosed cozero set $V$, and every $x \in \bar{V}-V=\partial V$, the measure $\hat{\mu}_{x}=$ evaluation at $x$ is supported in $\partial V$.

We use the following two fundamental results in the proof of the main theorem (Theorem 2.5).

Grothendieck Criterion. Suppose $S$ is a compact Hausdorff space and $B$ is a bounded subset of $M(S)$, the space of regular Borel measures on $S$. These are equivalent:

(a) $B$ is relatively $\sigma\left(M(S), M(S)^{*}\right)$ compact;

(b) for each pairwise disjoint sequence $\left\{G_{n}\right\}$ of open sets in $S$,

$$
\sup \left\{\left|\mu\left(G_{n}\right)\right|: \mu \in B\right\} \rightarrow 0 .
$$

For a proof, see [17, pp. 126-127] or [8, p. 227].

Rosenthal Theorem. Suppose $R$ is a continuous linear operator from $l^{\infty}$ into a Banach space $Y$ such that $\inf \left\{\left\|R\left(1_{\{i\}}\right)\right\|: i=1,2,3, \ldots\right\}>0$. Then there exists an infinite subset $\Gamma \subset \mathbf{N}$ such that $R \mid l^{\infty}(\Gamma)$ is an isomorphism.

For a proof, see $[15$, p. 17].

TheOREM 2.5. Suppose $S$ is a compact Hausdorff space, and the Banach lattice $L=C(S)$ satisfies the following two conditions:

(1) $L$ is order-Cauchy complete; and

(2) for every nonzero sequentially order continuous $\mu \geqslant 0$ in $L^{*}$, and every $f>0$ in $L$ with $\mu(f)=0$, there exists $g \in L$ such that $g \wedge f=0$ and $\mu(g)>0$.

Then for every nonweakly compact operator $T$ from $C(S)$ into a Banach space $Y$, there exists a Banach sublattice $L_{0} \subset C(S)$ such that $L_{0}$ is isometric as a Banach lattice to $l^{\infty}$ and $T \mid L_{0}$ is an isomorphism.

REMARK. Condition (2) of Theorem 2.5 is considerably illuminated by results of Veksler [24]. In particular, (2) is true if and only if (2a): every $\sigma$-normal measure (as in Proposition 2.1 above) annihilates every set which is the boundary of some zero set [24, Theorem 1.6]; and if and only if (2b): every $\sigma$-normal measure is "hereditarily $\sigma$-normal", in the sense that its trace on every closed set is $\sigma$-normal [24, Theorem 2.4]. Dualizing to vector lattice terminology, this requires that every 
sequentially order continuous functional on $C(S)$ must be $\left(o^{+}\right)$-linear, a formally stronger concept examined in [24].

Proof. Suppose $T: C(S) \rightarrow Y$ is a nonweakly compact operator into a Banach space $Y$. Then $B=T^{*}\left(\right.$ ball $\left.\left(Y^{*}\right)\right)$ is not relatively weakly compact in $C(S)^{*}$. By Grothendieck's criterion, there exist $\delta>0$, a sequence $\left\{\mu_{n}\right\} \subset B$, and a sequence $\left\{G_{n}\right\}$ of pairwise disjoint open sets in $S$, such that $\left|\hat{\mu}_{n}\left(G_{n}\right)\right|>\delta$ for all $n=$ $1,2,3, \ldots$ For each $n$, pick a compact $F_{n} \subset G_{n}$ with $\left|\hat{\mu}_{n}\right|\left(G_{n}-F_{n}\right)<\left|\hat{\mu}\left(G_{n}\right)\right|-$ $\delta$, and choose $f_{n} \in C(S)$ such that $f_{n}=1$ on $F_{n}, f_{n}=0$ off $G_{n}$, and $0<f_{n}<1$. Define $\nu_{n} \in C(S)^{*}$ by $\nu_{n}(g)=\left|\mu_{n}\right|\left(f_{n} g\right)$ for $g \in C(S)$ and all $n=1,2,3, \ldots$ Then $\left\|\nu_{n}\right\| \leqslant\left\|\mu_{n}\right\| \leqslant\left\|T^{*}\right\|=\|T\|$ for all $n$.

Let $\mu$ be any $w^{*}$ cluster point of $\left\{\nu_{n}\right\}$. Then $\mu \neq 0$ since for all $n, \nu_{n}(1)=\left|\mu_{n}\right|\left(f_{n}\right)$ $\geqslant\left|\hat{\mu}_{n}\right|\left(F_{n}\right) \geqslant\left|\hat{\mu}_{n}\left(G_{n}\right)\right|-\left|\hat{\mu}_{n}\right|\left(G_{n}-F_{n}\right)>\delta$. Furthermore, if an open set $G \subset S$ is disjoint from $\operatorname{coz}\left(f_{n}\right)$, then $\hat{\nu}_{n}(G)=0$, so that if $G$ intersects at most finitely many $\operatorname{coz}\left(f_{n}\right)$, then $\hat{\nu}_{n}(G)=0$ for almost all $n$, forcing $\hat{\mu}(G)=0$. Thus the support of $\hat{\mu}$ is contained in the boundary of the cozero set $\cup_{n=1}^{\infty} \operatorname{coz}\left(f_{n}\right)$. By the second hypothesis of the theorem, such $\mu \neq 0$ is not sequentially order continuous (use Lemma 2.2).

Consequently, choose $1 \geqslant g_{1} \geqslant g_{2} \geqslant \cdots \geqslant 0$ in $C(S)$ such that $\wedge_{i=1}^{\infty} g_{i}=0$ in the lattice $C(S)$, but for some $\varepsilon>0, \mu\left(g_{i}\right)>\varepsilon$ for all $i=1,2,3, \ldots$ Since $\mu$ is a $w^{*}$ cluster point of $\left\{\nu_{n}\right\}$, we can choose a subsequence $n_{1}<n_{2}<n_{3}<\ldots$ so that $\nu_{n_{i}}\left(g_{i}\right)=\left|\mu_{n_{i}}\right|\left(f_{n_{i}} g_{i}\right)>\varepsilon, i=1,2,3, \ldots$ Using the relation

$$
\begin{aligned}
\left|\mu_{n_{i}}\right|\left(f_{n_{i}} g_{i}\right) & =\sup \left\{\left|\mu_{n_{i}}(h)\right|:|h|<f_{n_{i}} g_{i}\right\} \\
& \leqslant 2 \sup \left\{\left|\mu_{n_{i}}(h)\right|: 0 \leqslant h \leqslant f_{n_{i}} g_{i}\right\}
\end{aligned}
$$

[17, p. 72] choose $h_{i} \in C(S)$ such that $0 \leqslant h_{i} \leqslant f_{n_{i}} g_{i}$ and $\left|\mu_{n_{i}}\left(h_{i}\right)\right|>\varepsilon / 2, i=$ $1,2,3, \ldots$ Finally, for each $i$, define $e_{i}=h_{i} /\left\|h_{i}\right\|$. These $e_{i}$ span a Banach sublattice of $C(S)$ isometric to $l^{\infty}$.

To verify this, note first that $\varepsilon / 2<\left|\mu_{n_{i}}\left(h_{i}\right)\right| \leqslant\left\|\mu_{n_{i}}\right\|\left\|h_{i}\right\| \leqslant\|T\|\left\|h_{i}\right\|$, so $\left\|h_{i}\right\|>$ $\varepsilon / 2\|T\|$ and $e_{i} \leqslant f_{n_{i}} g_{i} /\left\|h_{i}\right\| \leqslant 2\|T\| g_{i} / \varepsilon, i=1,2,3, \ldots$ Thus, for each $t \in l^{\infty}$ with $t \geqslant 0, t_{i} e_{i} \leqslant\|t\| 2\|T\| g_{i} / \varepsilon, i=1,2,3, \ldots$ Since $g_{i} \downarrow 0$ in the lattice $C(S)$, assumed to be up-down semicomplete, $\bigvee_{i=1}^{\infty} t_{i} e_{i} \equiv J^{+}(t)$ exists in $C(S)$ (see Theorem 1.5(2)). Since $e_{i} \wedge e_{j}=0$ for $i \neq j, J^{+}$is additive and positively homogeneous on the positive cone in $l^{\infty}$, so $J^{+}$extends uniquely to a positive linear map $J: l^{\infty} \rightarrow C(S)$. Clearly $J\left(t^{+}\right) \wedge J\left(t^{-}\right)=0$ for $t \in l^{\infty}$, so $J$ is a vector lattice homomorphism (see [17, pp. 58-59] for elementary properties of positive maps on vector lattices). Also, for $t \in l^{\infty}$,

$$
\|J(t)\|=\|J(|t|)\|=\left\|\bigvee_{i=1}^{\infty}\left|t_{i}\right| e_{i}\right\|=\sup \left|t_{i}\right|\left\|e_{i}\right\|=\|t\| .
$$

Thus $J$ is a Banach lattice isometry from $l^{\infty}$ into $C(S)$.

For the final step, observe that the map $R=T J$ from $l^{\infty}$ into $Y$ satisfies the condition of Rosenthal's theorem. In fact $\left\|R\left(1_{\{i\}}\right)\right\|=\left\|T\left(e_{i}\right)\right\| \geqslant\left|\mu_{n_{i}}\left(e_{i}\right)\right|>\left|\mu_{n_{i}}\left(h_{i}\right)\right|$ $>\varepsilon / 2$, by the choice of $h_{i}, i=1,2,3, \ldots$ Thus, for some infinite subset $\Gamma \subset \mathbf{N}$, $R \mid l^{\infty}(\Gamma)$ is an isomorphism. This just says that, for $L_{0}=J\left(l^{\infty}(\Gamma)\right) \subset C(S), T \mid L_{0}$ is 
an isomorphism. This $L_{0}$ is the desired Banach sublattice of $C(S)$ isometric to $l^{\infty}$. The proof is finished.

A Banach space $B$ is called a Grothendieck space if every $w^{*}$-convergent sequence in $B^{*}$ converges weakly, i.e. for $\sigma\left(B^{*}, B^{* *}\right)$; equivalently, every continuous linear operator from $B$ into $c_{0}$ is weakly compact (see Grothendieck [8, p. 229]). The conclusion of the foregoing Theorem 2.5 obviously implies that $C(S)$ is a Grothendieck space. Since this is a statement about weak convergence of sequences of measures, and since measures come defined on fields of sets or on Boolean algebras, it is useful to interpret these results for measures on a Boolean algebra. In fact, we obtain some additional information on boundedness of measures.

A bounded, finitely additive measure $\mu \geqslant 0$ on a Boolean algebra $A$ is called countably additive if $\mu\left(F_{n}\right) \rightarrow 0$ whenever $F_{1} \geqslant F_{2} \geqslant \ldots$ and $\wedge_{n=1}^{\infty} F_{n}=0$ in $A$. If $S$ is the Stone space of $A$, this is easily seen to be equivalent to requiring that the associated functional in $C(S)^{*}$ should be sequentially order continuous (see Proposition 2.1 above, and [19, p. 323], or [5, §44Cb, p. 113; §83Kh, p. 245]). The space of all bounded, finitely additive measures on the Boolean algebra $A$ is denoted $\mathrm{ba}(A)$.

TheOREM 2.6. Suppose $A$ is the Boolean algebra of closed-open sets in the compact totally disconnected space $S$, and suppose $A$ satisfies the following two properties:

(1) A is up-down semicomplete;

(2) for every nonzero bounded countably additive measure $\mu \geqslant 0$ on $A$, and every sequence $F_{1}, F_{2}, \ldots \in A$ with $\mu\left(F_{n}\right)=0$ for $n=1,2, \ldots$, there exists $G \in A$ with $G \wedge F_{n}=\varnothing$ for all $n$ and $\mu(G)>0$.

Then the conclusion of Theorem 2.5 holds for $C(S)$. Furthermore, if $\left\{\mu_{n}: n=\right.$ $1,2,3, \ldots\}$ is a sequence in $\mathrm{ba}(A)$ such that $\left\{\mu_{n}(F)\right\}$ is bounded (resp. converges) for each $F \in A$, then $\left\{\mu_{n}\right\}$ is norm bounded (resp. converges for the weak topology $\left.\sigma\left(\mathrm{ba}(A), \mathrm{ba}(A)^{*}\right)\right)$.

Proof. Condition (1) is equivalent to condition (1) of Theorem 2.5 (by Theorem 1.8 ) and the corresponding conditions (2) are easily seen to be equivalent. Thus the conclusion of Theorem 2.5 holds. The only part which needs proof is the boundedness assertion, for if $\left\{\mu_{n}\right\}$ is bounded and converges elementwise on $A$, then it converges on the dense subspace of $C(S)$ spanned by $\left\{1_{F}: F \in A\right\}$, so $\left\{\mu_{n}\right\}$ converges $w^{*}$ and hence weakly by the Grothendieck property. So assume $\left\{\mu_{n}(F)\right\}$ is bounded for each $F \in A$, but $\left\|\mu_{n}\right\| \rightarrow \infty$. Then there exist a disjoint sequence $\left\{F_{n}\right\}$ in $A$ and a subsequence of $\left\{\mu_{n}\right\}$, still called $\left\{\mu_{n}\right\}$, such that $1<\left|\mu_{n}\left(F_{n}\right)\right| \rightarrow \infty$ [18, Theorem 3.1] or [5, $\$ 81 \mathrm{~F}(\mathrm{v})$, p. 221]. Define measures $\nu_{n}$ on $A$ by $\nu_{n}(G)=$ $\left|\mu_{n}\right|\left(F_{n} \cap G\right) /\left|\mu_{n}\right|\left(F_{n}\right), G \in A$. Choose a $w^{*}$ cluster point $\mu$ of $\left\{\nu_{n}\right\}$, and exactly as in the proof of Theorem 2.5 except now using characteristic functions, construct a subsequence $\left\{F_{n_{i}}\right\}$ and subsets $E_{i} \subset F_{n_{i}}$ so that the functions $e_{i}=1_{E_{i}}$ are equivalent to the unit vectors in $l^{\infty}$. The isometry $J: l^{\infty} \rightarrow C(S)$ is given, as before, by $J(t)=\bigvee_{i=1}^{\infty} t_{i} e_{i}, t \geqslant 0$ in $l^{\infty}$. If $t=1_{M} \in l^{\infty}$ for some $M \subset \mathbf{N}$, then $J(t)=$ $\bigvee_{i \in M} e_{i}=1_{M^{\prime}}$, where $M^{\prime}=\bigvee_{i \in M} E_{i}$ in $A$. If we define $\gamma_{n}(M)=\mu_{n}\left(M^{\prime}\right), n=$ $1,2, \ldots$, then $\left\{\gamma_{n}(M)\right\}$ is bounded for all $M \subset \mathbf{N}$, but $\left\|\gamma_{n_{i}}\right\|=\left\|J^{*} \mu_{n_{i}}\right\| \rightarrow \infty$, 
violating the Phillips-Dieudonné boundedness theorem [15, p. 311]. This proves the theorem.

REMARK. There are many results in the literature dealing with uniform boundedness or weak convergence of sequences of finitely additive measures defined on $\sigma$-complete Boolean algebras, or on $\sigma$-fields of sets. Similarly, many theorems asserting various kinds of boundedness for vector measures assume that the domain is $\sigma$-complete. Many of these theorems can be extended to the algebras described in Theorem 2.6 (and to the fields of sets described in Theorem 3.6) because the important property is the convergence or boundedness assertion for scalar measures stated above. In particular, one can prove a Vitali-Hahn-Saks theorem for finitely additive measures defined on these algebras. The statement and proof would follow exactly the lines of the corresponding theorem of Seever for algebras whose Stone space is an $F$-space (see [18, Theorem 4.1]).

3. Baire-type sublattices of $\mathbf{R}^{X}$. In this section we describe a class of lattices of bounded functions to which the methods of $\$ \S 1$ and 2 apply (Theorem 3.4 below). We consider vector sublattices of $l^{\infty}(X) \subset \mathbf{R}^{X}$ for a point set $X$, i.e. bounded functions on $X$, where the (finite) lattice and algebra operations are defined pointwise on $X$. If $L$ is a sublattice of $l^{\infty}(X)$ then $L_{\delta}$ ("upper semi- $L$ ") is the family of all functions in $l^{\infty}(X)$ which are pointwise limits of decreasing sequences in $L$; $L_{\sigma}$ is similarly defined by increasing sequences.

Definition 3.1. If $L$ is a vector sublattice of $l^{\infty}(X)$ then $L$ is up-down pointwise closed if $L=L_{\sigma} \cap L_{\delta}$.

If $L$ is up-down semicomplete as a vector lattice then clearly $L$ is up-down pointwise closed, but the latter condition is much weaker. In fact, whenever $X$ is a topological space and $L=C(X)$ then $L$ is certainly up-down pointwise closed, but the up-down semicomplete $C(X)$ are quite special as shown in $\S 1$. Part of our effort here will be to give an additional condition on up-down pointwise closed $L$ to ensure that $L$ is up-down semicomplete, i.e., order-Cauchy complete, as a lattice (see Theorem 3.4 below).

Several characterizations of up-down pointwise closed $L$ emerge from the early studies of the Baire classes on $\mathbf{R}$. In order to summarize the known facts, we need some notation. If $L$ is a sublattice of $l^{\infty}(X)$, then $\Re a_{1}(L)$ denotes the first Baire class for $L$, i.e., the family of all $f \in l^{\infty}(X)$ which are pointwise limits on $X$ of sequences from $L$. We denote inductively for each ordinal $\alpha>1, \Re_{\alpha}(L)=$ $\mathscr{B a} \mathrm{a}_{1}\left(\cup_{\beta<\alpha} \mathscr{B a} \mathrm{a}_{\beta}(L)\right)$. The Baire system generated by $L$ is $\mathscr{B a}(L)=\mathscr{B a} a_{\omega_{1}}(L)$, the smallest family containing $L$ and closed under pointwise convergence of bounded sequences. By $\operatorname{coz}(L)$ we denote the family of all sets $\operatorname{coz}(f)=[f \neq 0] \subset X$ for $f \in L ; z(L)$ is all the sets $z(f)=[f=0] \subset X$ for $f \in L$. For a family $\mathscr{F}$ of subsets of $X, \mathscr{F}_{\sigma}$ (resp. $\mathscr{F}_{\delta}$ ) denotes the family of all unions (resp. intersections) of sequences from $\mathscr{F}$. If $\Sigma$ is a field of subsets of $X$ (i.e., $\Sigma$ contains $X$ and is closed under finite union and difference), then $l^{\infty}(X, \Sigma)$ denotes the uniformly closed linear subspace of $l^{\infty}(X)$ generated by characteristic functions of sets in $\Sigma$. The following theorem contains much of the classical information. 
THEOREM 3.2. Suppose $L$ is a vector sublattice of $l^{\infty}(X)$ containing the constants, and put

$$
\tilde{L}=\left\{f \in l^{\infty}(X): f^{-1}(G) \in \operatorname{coz}(L)_{\sigma} \text { for each open } G \subset \mathbf{R}\right\}
$$

Then:

(1) $\tilde{L}=L_{\sigma} \cap L_{\delta}$;

(2) if $f \in L_{\delta}, g \in L_{\sigma}$, and $f \leqslant g$, then $f \leqslant h \leqslant g$ for some $h \in \tilde{L}$;

(3) if $L=l^{\infty}(X, \Sigma)$ for some field $\Sigma$, then $\tilde{L}=l^{\infty}\left(X, \Sigma_{\sigma} \cap \Sigma_{\delta}\right)$;

(4) $\mathscr{B} \mathrm{a}_{1}(L)=\mathscr{B} \mathrm{a}_{1}(\tilde{L})=L_{\delta \sigma} \cap L_{\sigma \delta}=l^{\infty}\left(X, z(L)_{\delta \sigma} \cap \operatorname{coz}(L)_{\sigma \delta}\right)$.

These results go back essentially to Lebesgue and Hausdorff, with contributions from de la Vallé Poussin, Hahn, and Sierpinski. The classical theory in considerable generality, with appropriate literature citations, is presented in Hahn [10, Chapter IV and improvements on pp. 399-402]. Most of the methods and results, sometimes stated with unnecessary restrictions on $L$, are in Hausdorff [11, §41]. For a more recent exposition, see Mauldin [14]. The following corollary is immediate.

COROLlaRY 3.3. (1) $L$ is up-down pointwise closed if and only if $L=\tilde{L}$, and then $L$ is uniformly closed.

(2) If $L=l^{\infty}(X, \Sigma)$ for some field $\Sigma$, then $L$ is up-down pointwise closed if and only if $\Sigma=\Sigma_{\sigma} \cap \Sigma_{\delta}$ (i.e., $\Sigma$ is "up-down pointwise closed").

(3) For each ordinal $\alpha>0, \Re \mathrm{a}_{\alpha}(L)$ is up-down pointwise closed, uniformly closed, and has totally disconnected maximal ideal space.

The last assertion follows from the last representation of $\mathscr{B} \mathrm{a}_{1}(L)$ as a $l^{\infty}(X, \Sigma)$ in Theorem 3.2(4). See also Hahn [10, §31.4.52, p. 401].

REMARK. Lattices like these have come up quite recently in the work of Z. Frolik [6] and A. Hager [9]. Hager discusses some of the classical material stated above, and in addition gives several internal conditions on $L$ equivalent to up-down pointwise closure (e.g. relating to the existence of bounded inverses in $L$, or closure under Frolik's "strong continuous convergence").

We now state the main theorem of this section.

Theorem 3.4. Suppose $L$ is a vector sublattice of $l^{\infty}(X)$ containing the constants. Suppose also that there exists a vector sublattice $C$ of $L$ such that $C_{\delta} \subset L \subset \mathscr{B a}(C)$. If $L$ is up-down pointwise closed, then the sup-normed Banach lattice $L$ is representable as a $C(S)$ space which is order-Cauchy complete and satisfies the hypotheses of Theorem 2.5. Thus if $T$ is a nonweakly compact operator from $L$ into a Banach space, then $T$ restricts to an isomorphism on some subspace $L_{0} \subset L$ which is isometrically Banach-lattice isomorphic to $l^{\infty}$.

Proof. A functional $\mu \geqslant 0$ in $L^{*}$ is by definition sequentially smooth if $\mu\left(f_{n}\right) \rightarrow 0$ whenever $f_{1} \geqslant f_{2} \geqslant \ldots$ in $L$ and $f_{n}(x) \rightarrow 0$ for all $x \in X$. (Note that every sequentially order continuous $\mu \geqslant 0$ in $L^{*}$ is sequentially smooth, but the converse may in general fail because countable lattice infima in $L$ may be different from countable pointwise infima. One point to be shown here is that for $L$ as in this 
theorem, these two countable operations agree.) By the Daniell extension theorem (see [16, pp. 288-295]), each sequentially smooth $\mu \geqslant 0$ in $L^{*}$ has a unique extension to a sequentially smooth functional $\mu_{1}$ on $\mathscr{B a}(C)=\mathscr{B a}(L)$, and $\mu_{1}$ is determined by its values on $C_{\delta}$ by the formula

$$
\mu_{1}(h)=\sup \left\{\mu(g): 0 \leqslant g \leqslant h, g \in C_{\delta}\right\}, \quad h \geqslant 0 \text { in } \Re \mathrm{a}(L) .
$$

Now we verify that the lattice and pointwise countable suprema agree in $L$. Suppose $f_{1} \leqslant f_{2} \leqslant \ldots$ in $L$ and $\bigvee_{n=1}^{\infty} f_{n}=h$ exists in $L$, and let $f(x)=\sup f_{n}(x)$, $x \in X$. Then $f \leqslant h$ on $X$, and $h-f \in \mathscr{B a}(L)$. For each $x \in X$, the sequentially smooth functional $\delta_{x}=$ evaluation at $x$ satisfies $\delta_{x}(h-f)=h(x)-f(x)=$ $\sup \left\{g(x): 0 \leqslant g \leqslant h-f, g \in C_{\delta}\right\}$. But $C_{\delta} \subset L$, and if $g \in C_{\delta}$ with $0 \leqslant g \leqslant h-f$ then $f \leqslant h-g \leqslant h$ so $f_{n} \leqslant h-g \in L$ for all $n$. Since $h=\bigvee f_{n}$, then $g=0$. Thus $\delta_{x}(h-f)=0$ for $x \in X$, i.e., $h=f$. Similarly, lattice and pointwise countable infima agree in $L$. For such a vector lattice $L$, the notions of being up-down pointwise closed and of being up-down semicomplete, i.e., order-Cauchy complete, obviously are the same.

Finally, consider a sequentially order continuous $\mu \geqslant 0$ in $L^{*}$ and $f \geqslant 0$ in $L$ with $\mu(f)=0$. Then $\mu$ is sequentially smooth; let $\mu_{1}$ denote the Daniell extension of $\mu$ to $\mathscr{B a}(L)$. If $\mu \neq 0$, choose $h \geqslant 0$ in $L$ with $\mu(h) \neq 0$, and let $h_{0}$ be the "component of $h$ in $\mathscr{B a}(L)$ orthogonal to $f$ ", that is, $h_{0}=h-\bigvee_{n=1}^{\infty}(h \wedge n f)$ in $\mathscr{B a}(L)$. Then $h_{0} \wedge f=0$, and since $\mu_{1}$ is sequentially smooth, $\mu_{1}\left(\bigvee_{n=1}^{\infty}(h \wedge n f)\right)=$ $\sup _{n} \mu(h \wedge n f) \leqslant \sup \mu(n f)=0$. Thus $\mu_{1}\left(h_{0}\right)=\mu_{1}(h) \neq 0$, so there must exist $g \in$ $C_{\delta}$ such that $0 \leqslant g \leqslant h_{0}$ and $\mu(g)>0$. But then $g \in L$ and $g \wedge f \leqslant h_{0} \wedge f=0$, as required. Finally, the Banach lattice $L$ is an $M$-space with unit, so it is represented as a $C(S)$ for some compact space $S$ (see [17, p. 104]). This proves the theorem.

THEOREM 3.5. If $C$ is a vector sublattice of $l^{\infty}(X)$ containing the constants, and $\alpha>0$ is an ordinal, then the $\alpha$ th Baire class $L=\mathscr{B} \mathrm{a}_{\alpha}(C)$ is a Banach lattice under the sup norm which is representable as a $C(S)$ space which is order-Cauchy complete and satisfies the hypotheses of Theorem 2.5. Thus if $T$ is a nonweakly compact operator from $\mathscr{B}_{\mathrm{a}_{\alpha}}(C)$ into a Banach space then $T$ restricts to an isomorphism on some subspace $L_{0} \subset \mathscr{B a}_{\alpha}(C)$ which is isometrically Banach-lattice isomorphic to $l^{\infty}$.

Proof. $\Re \mathrm{a}_{\alpha}$ is up-down pointwise closed (Corollary 3.3(3)). Apply Theorem 3.4.

COROLlaRY. $\mathscr{B}_{\alpha}(C)$, or more generally any function lattice such as described in Theorem 3.4, is a Banach space which satisfies Grothendieck's property.

Proof. See the remarks after the proof of Theorem 2.5.

We interpret these results for measures. Denote by ba( $\Sigma)$ the space of all bounded finitely additive measures on a field of sets $\Sigma$.

THEOREM 3.6. Suppose $\Sigma$ is a field of subsets of $X$ and there exists a family $Z \subset \Sigma$ containing $X$ and $\varnothing$ such that $Z$ is closed under finite union and countable intersection, $F^{c} \in Z_{\sigma}$ for all $F \in Z$, and $Z$ generates the same $\sigma$-field as $\Sigma$ generates. If $\Sigma$ is up-down pointwise closed, i.e. if $\Sigma=\Sigma_{\sigma} \cap \Sigma_{\delta}$, then the Banach lattice $L=l^{\infty}(X, \Sigma)$ 
is order-Cauchy complete, and the conclusion of Theorem 2.5 holds for L. If $\left\{\mu_{n}\right\}$ is a sequence in $\mathrm{ba}(\Sigma)$ such that $\left\{\mu_{n}(F)\right\}$ is bounded (resp. converges) for each $F \in \Sigma$, then $\left\{\mu_{n}\right\}$ is norm bounded (resp. converges for the weak topology $\sigma\left(\mathrm{ba}(\Sigma), \mathrm{ba}(\Sigma)^{*}\right)$ ).

Proof. A $\mu \geqslant 0$ in $\mathrm{ba}(\Sigma)$ is by definition sequentially smooth if $\mu\left(F_{n}\right) \rightarrow 0$ whenever $F_{1} \supset F_{2} \supset \ldots$ in $\Sigma$ and $\cap F_{n}=\varnothing$. . y the extension theorem for measures [5, §71A, p. 193], every sequentially smooth $\mu>0$ in ba $(\Sigma)$ has a unique extension to a sequentially smooth measure $\mu_{1}$ on the $\sigma$-field $\Sigma_{1}$ generated by $\Sigma$, and $\mu_{1}$ is determined by its values on $Z \subset \Sigma$ by the formula $\mu_{1}(E)=$ $\sup \{\mu(F): F \subset E, F \in Z\}, E \in \Sigma_{1}$. Just as in the proof of Theorem 3.4, one applies this to point evaluation measures to deduce that countable (pointwise) unions and countable lattice suprema agree in $\Sigma$. Thus the Boolean algebra $A=\Sigma$ is up-down semicomplete. One again applies the extension theorem to a nonzero countably additive $\mu \geqslant 0$ in ba( $(\Sigma)$ to verify condition (2) of Theorem 2.6 , and the theorem follows.

EXAMPLE 3.7. The motivating example for fields $\Sigma$ of the type considered here is the class of all simultaneously $F_{\sigma}$ and $G_{\delta}$ subsets of a topological space $X$. Even for $X=[0,1]$, Theorem 3.6 is not obvious.

EXAMPLE 3.8. In case $S$ is a compact $F$-space, the proof that $C(S)$ is a Grothendieck space turns on the crucial fact that the support of every measure on $S$ is extremally disconnected (i.e. Stonian); see [18, p. 271] or [15, p. 20]. This method does not apply to the spaces considered here. We describe an example of a totally disconnected compact $S$ such that $C(S)$ satisfies Theorem 2.5 (so $C(S)$ is a Grothendieck space), yet the support of some measure on $S$ is not Stonian. Take $S$ to be the Stone space of the Boolean algebra $\Sigma$ of all simultaneously $F_{\sigma}$ and $G_{\delta}$ subsets of $[0,1]$ (Example 3.7), so that $C(S)$ represents the first Baire class on [0,1] (see [2]). Let $\mu$ be the measure induced on $S$ by Lebesgue measure restricted to $\Sigma$. Then, by the corollary to Theorem $3.5, C(S)$ is a Grothendieck space. To check that $\operatorname{supp}(\mu)$ is not Stonian, let $I_{1}, I_{2}, \ldots$ denote a base of open intervals in $[0,1]$ and construct by induction disjoint sets $F=\cup_{n=1}^{\infty} F_{n}, K=\cup_{n=1}^{\infty} K_{n}$, where $F_{n}$ and $K_{n}$ are closed, nowhere dense subsets of $I_{n}$ with positive measure. If $A \in \Sigma$ and $A$ almost contains $F$, in the sense that $F-A$ is a Lebesgue null set, then $F_{n} \cap A \neq \varnothing$ for all $n$, so $A$ is a dense $G_{\delta}$ in $[0,1]$. Likewise, if $A^{c}$ almost contains $K$ then $A^{c}$ is also a dense $G_{\delta}$ in $[0,1]$. By the category theorem, not both can occur. Thus $F$ and $K$ are represented in $\operatorname{supp}(\mu)$ by two disjoint open $F_{\sigma}$ sets whose closures intersect, so that $\operatorname{supp}(\mu)$ is not an $F$-space and therefore not Stonian.

EXAMPLE 3.9. In contrast to the preceding example, if $\Sigma$ is the field of subsets of $[0,1]$ generated by the closed sets, then $L=l^{\infty}([0,1], \Sigma)$ is definitely not a Grothendieck space; in fact $L=C(\Omega)$ for a compact $\Omega$ which contains a convergent sequence of distinct points (see [3]). Thus $L$ (and $\Sigma$ ) cannot be up-down pointwise closed (by Theorem 3.6).

\section{REFERENCES}

1. N. Aronszajn and P. Panitchpakdi, Extensions of uniformly continuous transformations and hyperconvex metric spaces, Pacific J. Math. 6 (1956), 405-439.

2. F. Dashiell, Isomorphism problems for the Baire classes, Pacific J. Matr. 52 (1974), 29-43.

3. ___ Weakly unconditionally summable sequences of continuous functions (preprint). 
4. F. Dashiell, A. Hager and M. Henriksen, Order-Cauchy completions of rings and vector lattices of continuous functions, Canad. J. Math. 32 (1980), 657-685.

5. D. H. Fremlin, Topological Riesz spaces and measure theory, Cambridge Univ. Press, Cambridge, 1974.

6. Z. Frolik, Three uniformities associated with uniformly continuous functions, Proc. Rome Conf. on Rings of Continuous Functions, 1973, Symposia Mathematica, vol. 17, Academic Press, New York, 1976, pp. 69-80.

7. L. Gillman and M. Jerison, Rings of continuous functions, Van Nostrand Reinhold, Princeton, N. J., 1960.

8. A. Grothendieck, Topological vector spaces, translated by O. Chaljub, Gordon and Breach, New York, 1973.

9. A. W. Hager, Real-valued functions on Alexandroff (zero-set) spaces, Comment. Math. Univ. Carolinae 16 (1975), 755-769.

10. H. Hahn, Reelle Funktionen, Chelsea, New York, 1948.

11. F. Hausdorff, Set theory, 2nd ed., Chelsea, New York, 1962.

12. M. Henriksen and D. Johnson, On the structure of a class of Archimedean lattice-ordered algebras, Fund. Math. 50 (1961), 73-94.

13. K. Kuratowski and M. Mostowski, Set theory, 2nd ed., North-Holland, Amsterdam, New York and Oxford, 1976.

14. R. D. Mauldin, Baire functions, Borel sets, and ordinary function system, Advances in Math. 12 (1974), 418-450.

15. H. P. Rosenthal, On relatively disjoint families of measures, with some applications to Banach space theory, Studia Math. 37 (1970), 13-36; correction, 311-313.

16. H. L. Royden, Real analysis, 2nd ed., Macmillan, New York, 1968.

17. H. H. Schaefer, Banach lattices and positive operators, Springer-Verlag, New York, 1974.

18. G. Seever, Measures on F spaces, Trans. Amer. Math. Soc. 133 (1968), 267-280.

19. Z. Semadeni, Banach spaces of continuous functions, PWN, Warsaw, 1971.

20. A. I. Veksler and V. A. Geiler, Order and disjoint completeness of linear partially ordered spaces, Sibirsk. Mat. Z. 13 (1972), 43-51 = Siberian Math. J. 13 (1972), 30-35.

21. C. J. Everett, Sequence completion of lattice moduls, Duke Math. J. 11 (1944), 109-119.

22. F. Papangelou, Order convergence and topological completion of commutative lattice-groups, Math. Ann. 155 (1964), 81-107.

23. J. Quinn, Intermediate Riesz spaces, Pacific J. Math. 56 (1975), 225-263.

24. A. I. Veksler, On one class of ordered sequentially continuous functions and regular Borel measures, Siberian Math. J. 17 (1976), 572-580.

Department of Mathematics, California institute of Technology, Pasadena, California 91125

Current address: 3303 Coolidge Avenue, Los Angeles, California 90066 